\title{
Treatment of Advanced Stage Gonarthrosis With Prolotherapy: Case Report
}

\author{
Ilker Solmaz ${ }^{1, *}$, Suleyman Deniz ${ }^{2}$, Onder Taylan Cifci ${ }^{3}$ \\ ${ }_{1}^{1}$ Turkey Proloteraphy and Pain Clinic, Istanbul, Turkey \\ ${ }_{3}^{2}$ Department of Anesthesiology, Gulhane Military Medical Academy, Haydarpasa Training Hospital, Istanbul, Turkey \\ ${ }^{3}$ Gumussuyu Military Hospital, Istanbul, Turkey \\ *Corresponding author: Ilker Solmaz, Turkey Proloteraphy and Pain Clinic, Istanbul, Turkey. Tel/Fax: +90-5327304814, E-mail: ilkersolmaz72@hotmail.com.
}

Received: November 18, 2012; Revised: September 28, 2013; Accepted: November 9, 2013

\begin{abstract}
Introduction: This case report aims to discuss the clinical and radiological outcomes of prolotherapy at a patient whom to total knee prosthesis had been planned but surgery couldn't be performed due to co-morbidities.

Case Presentation: A 72 year old woman presented with severe pain at her knees for over 5 years. Treatment methods include weight loss, decreasing the weight bearing on the joint, stretching exercises, nonsteroid anti-inflammatory and steroid drugs, and physiotherapy. The Western Ontario and McMaster Universities Osteoarthritis Index (WOMAC) scale was applied to measure the osteoarthritis level of the patient: Pain level; 25 points, stiffness level; 10 points, Physical function loss; 80 points, and total WOMAC 115 points. At radiological evaluation, the patient was diagnosed as grade IV osteoarthritis due to significant osteophyte presence and complete joint space narrowing. Six sessions of knee prolotherapy protocol was applied to the patient, one session monthly.

Conclusions: Significant improvement was noted at WOMAC scale (Pain level; 5 points, stiffness level; 2 points, Physical function loss; 15 points, and total WOMAC 22 points). Osteoarthritis level of the patient was improved to grade I at radiological evaluation after a year. Our case is the report that presents radiological evidence in addition to clinical findings of improvement of osteoarthritis level. As a result of this case report, further studies aiming to offer a different minimally invasive treatment option to the patients before surgery may be performed. We are in the opinion that prolotherapy may be preferred more commonly as an efficient method once the importance of ligamentous structures at pathogenesis of osteoarthritis is established.
\end{abstract}

Keywords: Osteoarthritis, Knee; Degeneration; Treatment

\section{Introduction}

Degenerative joint disease (osteoarthritis) is a chronic, non-inflammatory and common joint disease characterized with degeneration of synovial joint cartilage, and new bone formation at joint surfaces and margins (1).

Osteoarthritis is the most common joint disease and one of the most common causes of physical deformity. It affects both genders and all races. Although $30 \%$ of individuals above 75 years old have symptoms, non-symptomatic (radiologic) osteoarthritis is presented over $20 \%$ of the patients at 3 rd decade and $80 \%$ at 8 th decade (2).

Knee is the most commonly affected joint, particularly due to weight bearing. Knee osteoarthritis may significantly impact an individual's quality of life; even make walking impossible (2).

Treatment methods include weight loss, decreasing the weight bearing on the joint, stretching exercises, nonsteroid anti-inflammatory and steroid drugs, and physiotherapy. On the other hand, a pathological process including exchange of joint surface with metals may be preferred at orthopedic approach $(1,2)$.

Prolotherapy is an injection therapy method that is ca- pable of reversing the degeneration process by activating the regeneration potential of the body. This is the only method capable of avoiding the patient from major surgical procedures by providing clinical improvement at even grade IV gonarthrosis (3).

This case report aims to discuss the clinical and radiological outcomes of prolotherapy at a patient whom to total knee prosthesis had been planned but surgery couldn't be performed due to co-morbidities.

\section{Case Presentation}

A 72 year old woman presented with severe pain at her knees for over 5 years. She had history of chronic obstructive pulmonary disease for 10 years, diabetes mellitus for 20 years, and hypertension for 30 years. In addition to physiotherapy, intra-articular steroid two years ago and hyaluronic acid injections one year ago had been applied, and her complaints had not resolved with these treatments, although she used $500 \mathrm{mg}$ paracetamol, $30 \mathrm{mg}$ caffeine, 10 mg codein every six hour and 25 mg dexketoprofen trometamol every eight hour.

The last physician patient was referred had recom-

Implication for health policy/practice/research/medical education:

Prolotraphy treatment offer different minimally invasive treatment option to the patients whom surgery could not performed due to co-morbidities.

Copyright @ 2013, Iranian Society of Regional Anesthesia and Pain Medicine (ISRAPM); Published by Kowsar Corp. This is an open-access article distributed under the terms of the Creative Commons Attribution License, which permits unrestricted use, distribution, and reproduction in any medium, provided the original work is properly cited. 
mended total knee prosthesis but stated that surgical intervention would be unfavorable due to her irregular chronic diseases. After all, the patient was referred to our clinic.

Physical examination revealed tenderness at medial and lateral collateral ligaments, pes anserius, patellar ligament, and coronary ligament. There was a decrease at joint flexion angle, range of motion ( $\mathrm{ROM}=90$ degree) and stress tests were positive.

Visual Analog Scale (VAS), established by Price et al. (4), was used to measure the pain level of the patient. Pain level was detected as close to the most severe pain level (Scala 1).

The Western Ontario and McMaster Universities Osteoarthritis Index (WOMAC) scale (5) was applied to measure the osteoarthritis level of the patient: Pain level; 25 points, stiffness level; 10 points, Physical function loss; 80 points, and total WOMAC 115 points.

At radiological evaluation, the patient was diagnosed as grade IV osteoarthritis due to significant osteophyte presence and complete joint space narrowing (Table 1) (Figure 1).

Blood analysis and biochemical tests were reported as normal. Administration of prolotherapy protocol was decided after getting the written informed consent from the patient.

\subsection{Therapy Protocol}

Six sessions of knee prolotherapy protocol was applied to the patient, one session monthly.

The patient was monitored with ECG, arterial blood pressure and pulse oximeter measurements. 50 mcg fentanyl, $1 \mathrm{mg}$ midazolam, and $50 \mathrm{mg}$ propofol were given to the patient in divided doses for sedoanalgesia.

Tenderness points at examination were marked after sterilizing the injection area. 4 cc of $25 \%$ dextrose $+4 \mathrm{cc}$ of $0.2 \%$ lidocaine solution was injected intra-articularly. Lateral knee injections.

Table 1. Radiological Knee Staging of Gonarthrosis Patients

\begin{tabular}{ll}
\hline Stage & Explanation \\
\hline $\mathbf{1}$ & Minimal osteophyte, normal joint space \\
$\mathbf{2}$ & $\begin{array}{l}\text { Significant osteophyte, suspicious joint space nar- } \\
\text { rowing }\end{array}$ \\
$\mathbf{3}$ & $\begin{array}{l}\text { Significant osteophyte and significant joint space } \\
\text { narrowing }\end{array}$ \\
$\mathbf{4}$ & $\begin{array}{l}\text { Significant osteophyte and complete joint space } \\
\text { narrowing }\end{array}$ \\
\hline
\end{tabular}

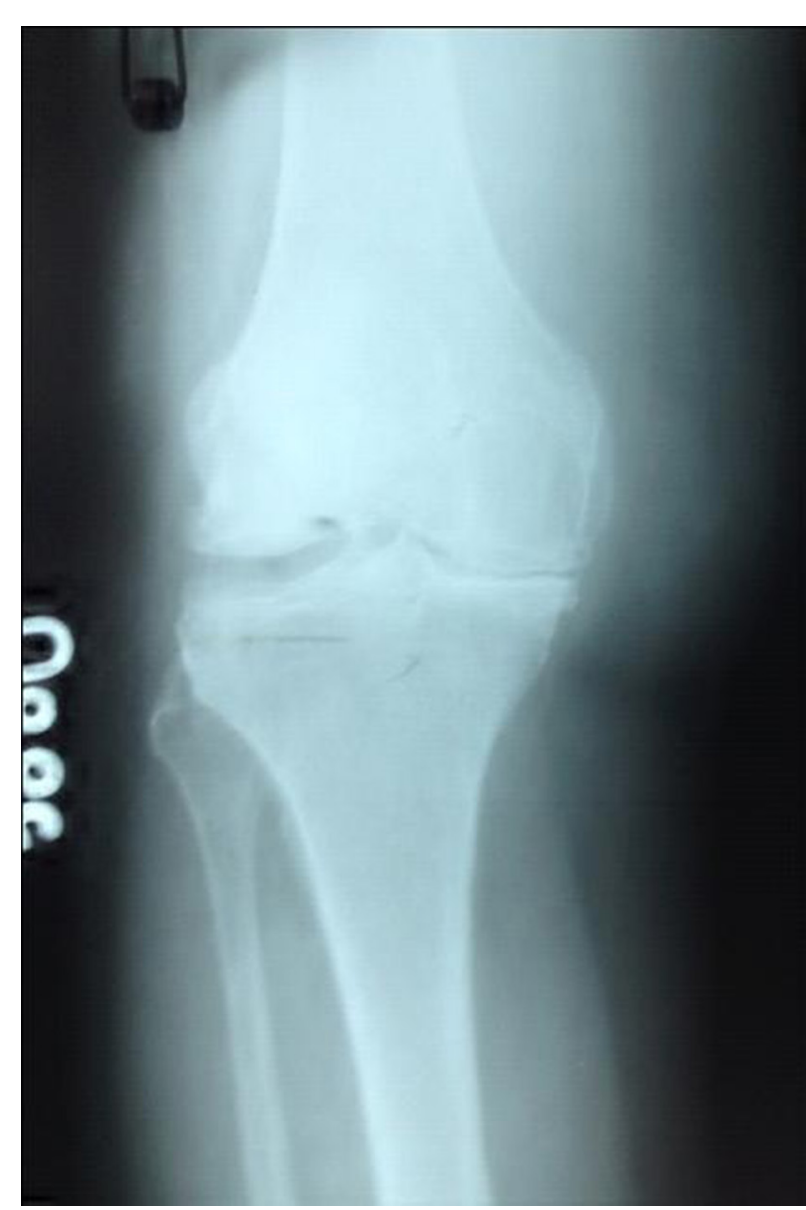

Figure 1. Knee Radiography Before Prolotherapy

0.5 cc of $15 \%$ dextrose +0.5 cc of $0.2 \%$ lidocaine solution was injected to per certain points around the joint (joint capsule, insertion of medial coronary ligament, insertion of medial collateral ligament, teno-periosteal junction, insertion of pes anserious semimembranosus point over tibia, insertion of gastrocnemius and adductor magnus points over femur, fibro-osseous junction, insertion of arcuate and oblique ligaments, insertion of lateral coronary ligament, insertion of lateral collateral ligament, teno-periosteal junction, iliotibial tract at tibia, insertion of biceps femoris point at fibular head, insertion of gastrocnemius and popliteus points at femur).

We administered five times the skin attempt for 15 point injections and used 22 cc solutions totally.

Nonsteroidal anti-inflammatory drugs and steroids have been lost. Weight loss, decreasing the weight bearing on the joint, stretching exercises and physiotherapy was continued. 


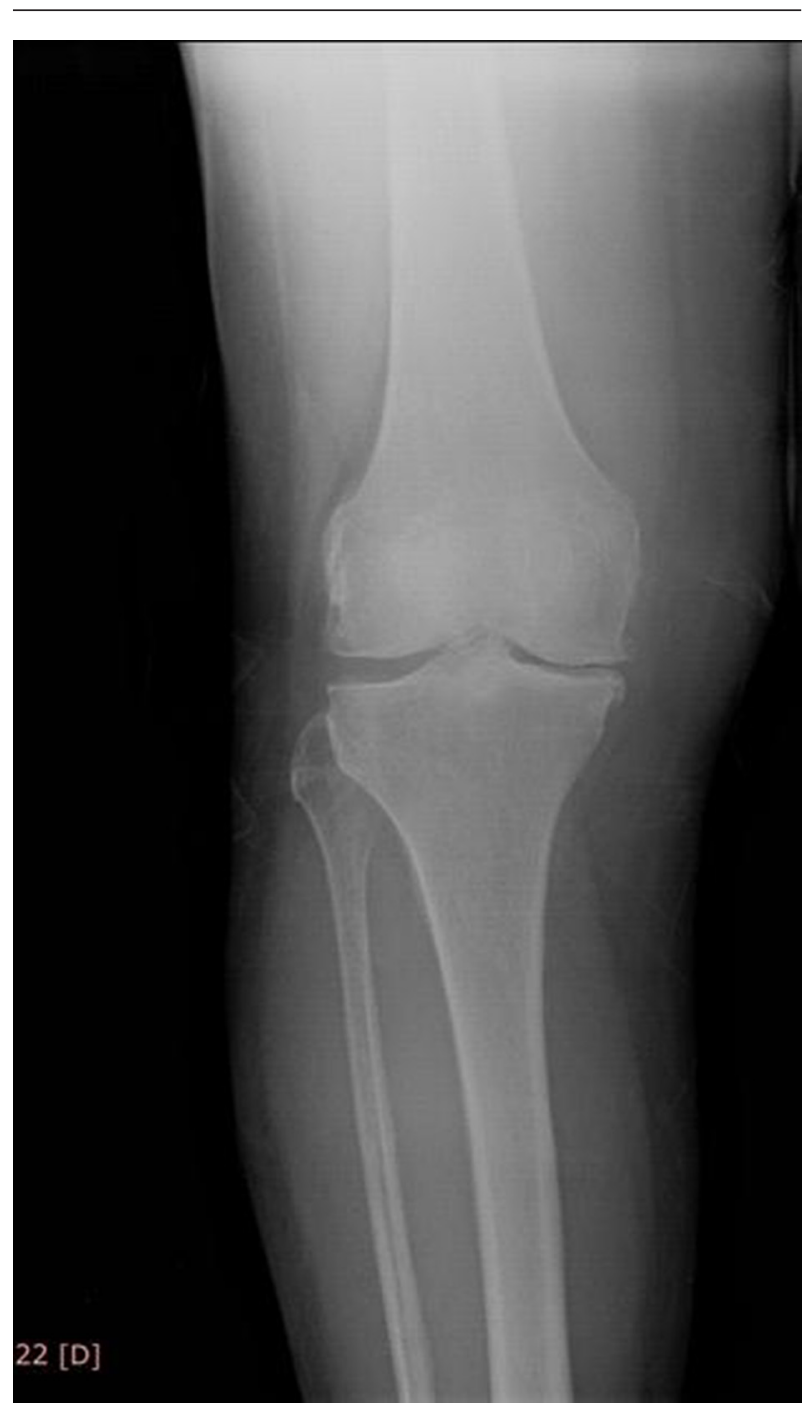

Figure 2. Knee Radiography After Prolotherapy

\section{Conclusions}

The patient was evaluated at the end of 6 sessions. VAS score was measured as close to the no-pain point (Scala 2).

Significant improvement was noted at WOMAC scale (Pain level; 5 points, stiffness level; 2 points, Physical function loss; 15 points, and total WOMAC 22 points).

Osteoarthritis level of the patient was improved to grade I at radiological evaluation after a year (Figure 2 ).

There was a increase at joint flexion angle, range of motion ( $\mathrm{ROM}=115$ degree $)$.

It is quite important that an improvement was shown at a patient with advanced stage gonarthrosis by clinical and radiological evidences after prolotherapy. In addition to the decrease at pain levels, grade IV arthritis was radiographically improved to grade I after a year.
Injection therapies are commonly used for gonarthrosis as non-surgical treatment methods. The majority of these procedures include either corticosteroids or hyaluronic acid. These therapies decrease the complaints at short-term. But no evidence could be found that they improve arthritis level at long-term (1-3, 6, 7). Clinical and radiological improvements were demonstrated with our therapy method.

A limited number of double-blinded, randomized studies are found in the literature. The study by Hackett et al. (6) in 1960 is the first review. The studies of Reeves et al. (8-10) are the other studies demonstrating evidence of significant clinic improvements at patients after prolotherapy.

Prolotherapy is a procedure which proliferative solutions are injected into ligamentous structures for regeneration. Injected solution causes inflammation at connective tissue. The immune response to inflammation regenerates ligamentous compounds and resolves ligamentous laxity occurring at joint during arthritis $(6,7)$.

The reason of degeneration at synovial joint cartilage which has role at the pathology of osteoarthritis has not been well-established yet $(6,7)$. In our case, prolotherapy has been successful at reversal of the degeneration. Thus, ligamentous structures may be considered to have a key role at formation of arthritis. The stability of the joint is maintained by ligamentous structures. The impairment at joint mechanics due to laxity and degeneration at these structures may cause excessive weight bearing at synovial tissues. Cartilage degeneration, a reason for the complaints, may be the last step of the degeneration at pathological pathway; not the onset of the arthritis (7).

The reason that therapies concerning only synovial cartilage provide only temporary improvements is these therapies don't strengthen important ligamentous structures. The improvement at joint mechanics after regeneration of ligamentous structures may result with synovial regeneration (7). Ligamentous approach may offer new solutions at the treatment of arthritis and prevent plenty of patients from major surgical procedures.

Our case is the report that presents radiological evidence in addition to clinical findings of improvement of osteoarthritis level.

As a result of this case report, further studies aiming to offer a different minimally invasive treatment option to the patients before surgery may be performed.

We are in the opinion that prolotherapy may be preferred more commonly as an efficient method once the importance of ligamentous structures at pathogenesis of osteoarthritis is established.

\section{Acknowledgements}

The authors are grateful to Department of Anesthesiology, Gulhane Military Medical Academy, Haydarpasa Training Hospital doctors for their cooperation. 


\section{Authors' Contribution}

Study concept and design: Ilker Solmaz, Suleyman Deniz, Onder Taylan Cifci, Acquisition of data: Ilker Solmaz, Suleyman Deniz, Onder Taylan Cifci, Analysis and interpretation of data: Ilker Solmaz, Onder Taylan Cifci , Drafting of the manuscript: Ilker Solmaz, Onder Taylan Cifci , Critical revision of the manuscript for important intellectual content: Ilker Solmaz, Suleyman Deniz, Onder Taylan Cifci , Statistical analysis: Ilker Solmaz, Suleyman Deniz, Administrative, technical, and material support: Ilker Solmaz, Suleyman Deniz, Study supervision: Ilker Solmaz, Suleyman Deniz, Onder Taylan Cifci .

\section{Financial Disclosure}

We certify that there is no conflict of interest with any financial organization regarding the material discussed in the manuscript.

\section{Funding/Support}

The research presented in this manuscript has not been funded by any institution.

\section{References}

1. McAlindon TE, Cooper C, Kirwan JR, Dieppe PA. Determinants of disability in osteoarthritis of the knee. Ann Rheum Dis. 1993;52(4):258-62.

2. Kellgren JH, Lawrence JS. Radiological assessment of rheumatoid arthritis. Ann Rheum Dis. 1957;16(4):485-93.

3. Rabago D, Best TM, Beamsley M, Patterson J. A systematic review of prolotherapy for chronic musculoskeletal pain. Clin J Sport Med. 2005;15(5):376-80.

4. Price DD, McGrath PA, Rafii A, Buckingham B. The validation of visual analogue scales as ratio scale measures for chronic and experimental pain. Pain.1983;17(1):45-56.

5. Stratford PW, Kennedy DM, Woodhouse LJ, Spadoni GF. Measurement properties of the WOMAC LK 3.1 pain scale. Osteoarthritis Cartilage. 2007;15(3):266-72.

6. Hackett GS. Prolotherapy in whiplash and low back pain. Postgrad Med.1960;27:214-9.

7. Hackett GS, Huang TC, Raftery A, Dodd TJ. Back pain following trauma and disease--prolotherapy. Mil Med.1961;126:517-25.

8. Reeves KD, Hassanein K. Randomized prospective double-blind placebo-controlled study of dextrose prolotherapy for knee osteoarthritis with or without ACL laxity. Altern Ther Health Med. 2000;6(2):68-74.

9. Reeves KD, Hassanein K. Randomized, prospective, placebocontrolled double-blind study of dextrose prolotherapy for osteoarthritic thumb and finger (DIP, PIP, and trapeziometacarpal) joints: evidence of clinical efficacy. J Altern Complement Med. 2000;6(4):311-20.

10. Reeves KD, Hassanein KM. Long-term effects of dextrose prolotherapy for anterior cruciate ligament laxity. Altern Ther Health Med. 2003;9(3):58-62. 